\title{
Thermo-mechanical stress analysis of dissimilar material joints using FEM
}

Somnath SOMADDER

DOI: 10.30464/jmee.2020.4.2.147

Cite this article as:

Somadder S. Thermo-mechanical stress analysis of dissimilar material joints using FEM. Journal of Mechanical and Energy Engineering, Vol. 4(44), No. 2, 2020, pp. 147-154.

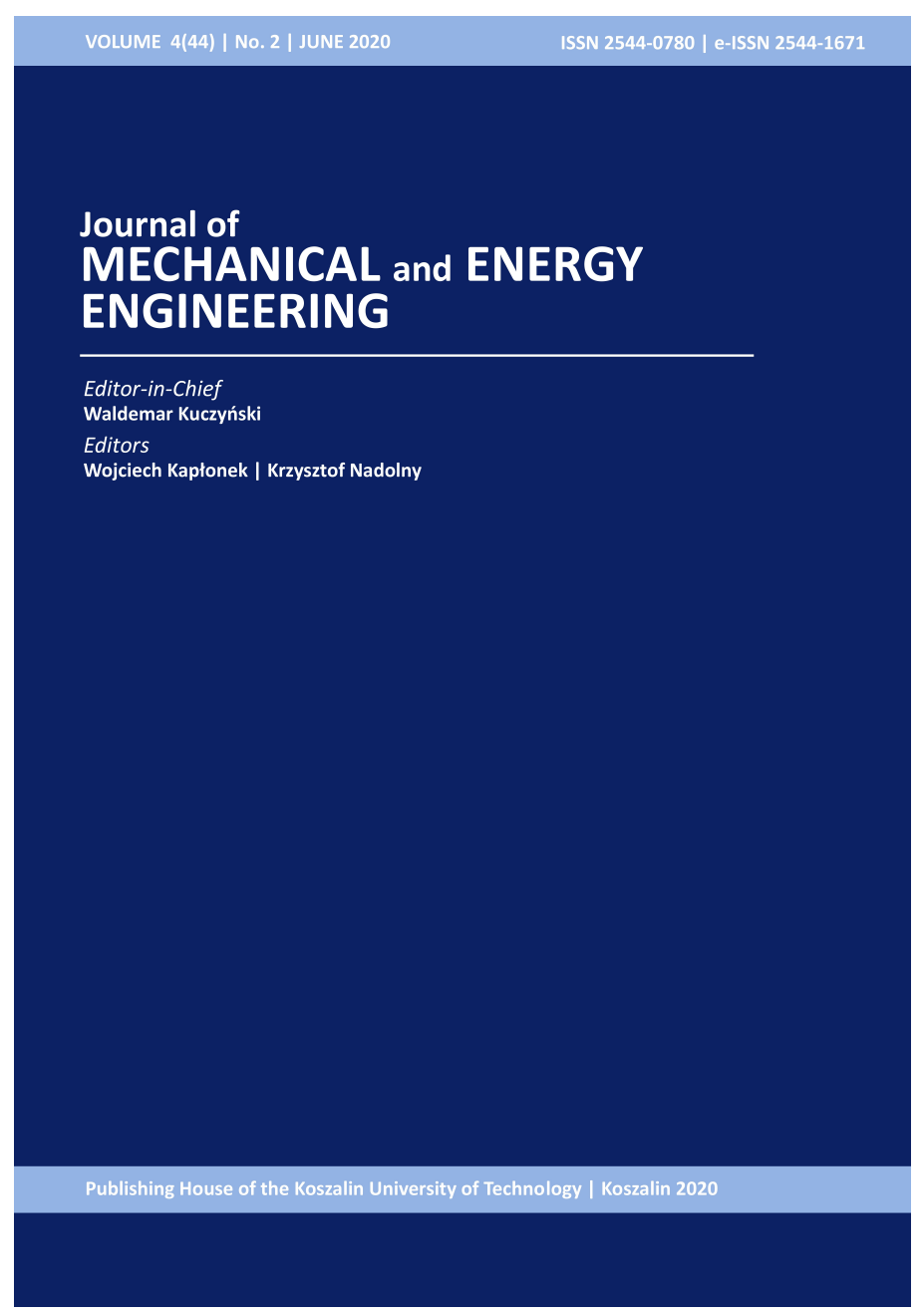

Journal of Mechanical and Energy Engineering

Website: jmee.tu.koszalin.pl

ISSN (Print): 2544-0780

ISSN (Online): 2544-1671

Volume: 4(44)

Number: 2

Year: 2020

Pages: $147-154$

Article Info:

Received 3 June 2020

Accepted 9 July 2020

Open Access

This article is distributed under the terms of the Creative Commons Attribution 4.0 (CC BY 4.0) International License (http://creativecommons.org/licenses/by/4.0/), which permits unrestricted use, distribution, and reproduction in any medium, provided you give appropriate credit to the original author(s) and the source, provide a link to the Creative Commons license, and indicate if changes were made. 


\title{
THERMO-MECHANICAL STRESS ANALYSIS OF DISSIMILAR MATERIAL JOINTS USING FEM
}

\author{
Somnath SOMADDER ${ }^{1 *}$ \\ 1* Faculty of Mechanical Engineering, Department Mechanical Engineering, \\ Khulna University of Engineering \& Technology, Bangladesh, e-mail: somnath@me.kuet.ac.bd
}

(Received 3 June 2020, Accepted 9 July 2020)

\begin{abstract}
This article presents numerical investigation of isotropic dissimilar material joints. Dissimilar material joints are broadly used in in various structures, including offshore, nuclear, electronic packaging, IC chip and spacecraft various fields of science and technology. In bimaterial joints two different material are bonded with common interface region. High stress concentration occur at the interface of the joint under thermo-mechanical loadings due to the difference in the elastic properties and the thermal expansion coefficients of dissimilar materials. The stresses acting along the interface of dissimilar material joints are very important to determine whether the structure is reliable or not for operation. The main purpose of this research is to provide finite element solutions to predict the stress distribution at the interface of the joint based on the theory of elasticity.
\end{abstract}

Keywords: Numerical Investigation, Dissimilar material joints, Stress concentration, Stress distributions, Theory of elasticity.

\section{INTRODUCTION}

Recently finite element analysis have become the most convenient analyst's tool and have dealt with the real world of design engineering. Now CAD software has built-in FEA capabilities and researchers use FEA as an everyday design tool in support of the product design process.

In IC chip including SIM card bonded joints are used. Bonded joint refers to the joint in which two or more materials are joined by means of adhesive. When mechanical or thermal load acts on IC chip, large stress develops in the interface, actually near the vertex causing the bonded joint to deboned damaging the component. For this reason the electronic circuit is failed.

Strength of bi-material joint greatly depends on the orientation of physical properties and material structure. These geometrical parameters affect the performances of a bonded joint. These discontinuities may cause singularities in the stress fields or very stress concentration near the vertex of the bonding edges. This stress concentration/singularity may lead to the delamination initiation in the local area, and subsequently to the global failure of the joint structures [1].
Previously many analysis have done in the literature of the dissimilar joints. Several research studies have been conducted and reported to determine permissible stress levels, criteria of failure and material behavior at the interface of bi metallic joint. Hideo Koguchi analyzed stress singularity at three dimensional bonded joints [2]. Somnath Somadder and Md. Shahidul Islam investigated stress and displacement field of cylinder subjected to thermomechanical loadings by using finite element method [3]. A. Barut, I. Guven and E. Madenci determined singular stress field at multiple dissimilar material joints subjected to mechanical and thermal loading [4]. Hideo Koguchi and M. Nakajima investigated the variation of intensity of singular stress field with interlayer thickness in three-dimensional three-layered joint due to external load using boundary element method [5]. Hideo Koguchi, Yasuyuki Tsukada and Takahiko Kurahashi analyzed three dimensional singular strain field employing digital image correlation method near the corner of SI chip [6]. C. Luangarpa and Hideo Koguchi investigated 3D dissimilar material bonded joints using conservative integral one real singularity [7]. Somnath Somadder and Md. Shahidul Islam investigated stress field of a thick walled orthotropic bonded cylinder under 
pressure and temperature [8]. D. Munz, A. Matthias and Y. Y. Yang determined thermal stresses in ceramic-metal joint having an inter-layer [9]. $\mathrm{H}$. Pengfei, H. Ishikawa and Y. Kohno analyzed order of stress singularity at the corner of a diamond shaped rigid inclusion under bending [10].

\section{ANALYTICAL SOLLUTION}

It is a law of nature that "matter change shapes by temperature changes." For most materials, "Increase temperature will make its shape larger, and the reverse is true too." Almost all metals expand with temperature increase, and contract with decrease of temperature. In general temperature, or temperature changes in a solid may induce the following effects.

1. Temperature increase will change material properties: Such as decrease the Young's modulus and yield strength of materials.

2. Induce thermal stresses that will be added to mechanically induced stresses in solid structures.

3. Induce creep of the material, and there by make materials vulnerable for failure at high temperature.

\subsection{Causes of thermal stress}

There are two causes of thermal stresses in solid structures.

\section{A uniform temperature}

A uniform temperature increase in a solid rod with both ends fixed will induce compressive stress in the rod with an amount equal to:

$$
\sigma=-\alpha E \Delta T
$$

where, $\alpha=$ the coefficient of thermal expansion with a unit of $I^{\circ} \mathrm{C}, \Delta T=$ temperature rise from a reference temperature, $\mathrm{E}=$ Modulus of elasticity of the material.

\section{Solid with non-uniform temperature distributions}

Stress induced by non-uniform temperature distributions of solid cause internal restraints for thermal expansion or contractions.

For two dimensional thermal stress problem, there will be two normal strains $\varepsilon_{x T}$ and $\varepsilon_{y T}$ along with a shear strain $\gamma_{x y T}$ because of different mechanical properties in the $\mathrm{x}$ and $\mathrm{y}$ directions for anisotropic materials. The thermal strain matrix for anisotropic material is then:

$$
\varepsilon_{T}=\left\{\begin{array}{c}
\varepsilon_{x T} \\
\varepsilon_{y T} \\
\gamma_{x y T}
\end{array}\right\} .
$$

For the case of plane stress in an isotropic material with coefficient of thermal expansion $\alpha$ subjected to temperature rise $T$. The thermal strain matrix becomes:

$$
\varepsilon_{T}=\left\{\begin{array}{c}
\alpha T \\
\alpha T \\
0
\end{array}\right\} .
$$

No shear strains caused by a change in temperatures of isotropic materials, only expansion or contraction.

For this case plane strain in an isotropic material, the thermal strain matrix is:

$$
\varepsilon_{T}=(1+v)\left\{\begin{array}{c}
\alpha T \\
\alpha T \\
0
\end{array}\right\} .
$$

For a constant thickness and constant triangular element the thermal force matrix can be obtained such as:

The total strain energy is given as:

$$
U=\int_{V} u_{0} d V .
$$

For a simple one dimensional rod thermal strain:

$$
\varepsilon_{x}=\frac{\sigma_{x}}{E}+\varepsilon_{T} .
$$

Let, $\frac{1}{E}=D^{-1}$ then in general matrix form equation can be written as:

$$
\underline{\varepsilon}=[D]^{-1} \underline{\sigma}+\underline{\varepsilon}_{T} .
$$

We solve for $\sigma$ as

$$
\underline{\sigma}=\underline{D}\left(\underline{\varepsilon}-\underline{\varepsilon}_{T}\right) .
$$

The strain energy per unit volume

$$
\begin{gathered}
u_{0}=\frac{1}{2} \underline{\sigma}\left(\underline{\varepsilon}-\underline{\varepsilon}_{T}\right), \\
\therefore u_{0}=\frac{1}{2}\left(\underline{\varepsilon}-\underline{\varepsilon}_{T}\right)^{T} \underline{D}\left(\underline{\varepsilon}-\underline{\varepsilon}_{T}\right) .
\end{gathered}
$$

The transpose is needed on the strain matrix to multiply matrices properly. Now:

$$
U=\frac{1}{2} \int_{V}\left(\underline{\varepsilon}-\underline{\varepsilon}_{T}\right)^{T} \underline{D}\left(\underline{\varepsilon}-\underline{\varepsilon}_{T}\right) d V .
$$

Using $\underline{\varepsilon}=\underline{B} \underline{d}$ in equation (11):

$$
U=\frac{1}{2} \int_{V}\left(\underline{B} \underline{d}-\underline{\varepsilon}_{T}\right)^{T} \underline{D}\left(\underline{B} \underline{d}-\underline{\varepsilon}_{T}\right) d V .
$$

Simplifying(12)

$$
U=\frac{1}{2} \int_{V}\left(\underline{d}^{T} \underline{B}^{T} \underline{D} \underline{B} \underline{d}-\underline{d}^{T} \underline{B}^{T} \underline{D}_{T}-\underline{\varepsilon}_{T}^{T} \underline{D} \underline{B} \underline{d}+\underline{\varepsilon}_{T}^{T} \underline{D} \underline{\varepsilon}_{T}\right) d V .
$$

The first term in the equation (13) is the strain energy due to stress produced from mechanical loading - that is: 


$$
U_{L}=\frac{1}{2} \int_{V} \underline{d}^{T} \underline{B}^{T} \underline{D} \underline{B} \underline{d} d V .
$$

Terms 2 and 3 in equation (13) are identical and can be written together as:

$$
U_{T}=\frac{1}{2} \int_{V} \underline{d}^{T} \underline{B}^{T} \underline{D} \underline{\varepsilon}_{T} d V .
$$

The last and fourth term is a constant and drops out when principal of minimum potential energy is applied by setting:

$$
\frac{\partial U}{\partial \underline{d}}=0 .
$$

Therefore letting $U=U_{T}+U_{L}$ and substituting equation (14) and (15) into (16) we obtain:

$$
\begin{gathered}
\frac{\partial U_{L}}{\partial \underline{d}}=\int_{V} \underline{B}^{T} \underline{D} \underline{B} d d V, \\
\frac{\partial U_{T}}{\partial \underline{d}}=\int_{V} \underline{B}^{T} \underline{D}_{T} d V=\left\{f_{T}\right\} .
\end{gathered}
$$

Recalling equation (3):

$$
\left[\because\left\{\varepsilon_{T}\right\}=\alpha T\right] .
$$

Thermal force matrix,

$$
\left\{f_{T}\right\}=A \int_{0}^{L}[B][D]\{\alpha T\} d x .
$$

For a constant thickness (t), constant-strain triangular element equation (20) can be simplified for two dimensional case as:

$$
\left\{f_{T}\right\}=[B]^{T}[D]\left\{\varepsilon_{T}\right\} t A .
$$

By substituting equation for $[D]$ and $[B]$ as follows:

$$
\begin{gathered}
{[D]=\frac{E}{1-v^{2}}\left[\begin{array}{ccc}
1 & v & 0 \\
v & 1 & 0 \\
0 & 0 & \frac{1-v}{2}
\end{array}\right],} \\
{[B]=\left[\begin{array}{lll}
B_{i} & B_{j} & B_{m}
\end{array}\right],}
\end{gathered}
$$

Thermal force matrix becomes:

$$
\left\{f_{T}\right\}=\frac{\alpha E t T}{2(1-v)}\left\{\begin{array}{c}
\beta_{i} \\
\gamma_{i} \\
\beta_{j} \\
\gamma_{j} \\
\beta_{m} \\
\gamma_{m}
\end{array}\right\} .
$$

\subsection{Stress/Strain Relationships}

Three dimensional stress/strain relationships for an isotropic body will be developed. This is done by considering the response of a body imposed stresses. The body is subjected to stresses $\sigma_{x}, \sigma_{y}$ and $\sigma_{z}$ independently as shown in figure.

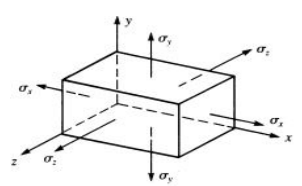

(a)

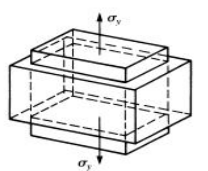

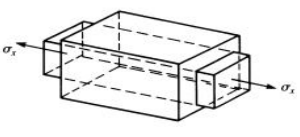

(b)

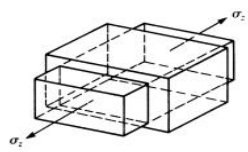

Fig. 1. Element subjected to normal stress acting in three mutually perpendicular directions

Consider, the stress in the $\mathrm{X}$ direction produces a positive strain:

$$
\varepsilon_{x}^{\prime}=\frac{\sigma_{x}}{E} .
$$

The positive stress in the $\mathrm{y}$ direction produces a negative strain in the $\mathrm{x}$ direction as a result of Poisson's effect given by:

$$
\varepsilon_{x}^{\prime \prime}=-\frac{v \sigma_{y}}{E} .
$$

The positive stress in the $\mathrm{z}$ direction produces 0) a negative strain in the $\mathrm{x}$ direction as a result of Poisson's effect given by:

$$
\varepsilon_{x}^{\prime \prime \prime}=-\frac{v \sigma_{z}}{E} .
$$

Using superposition we obtain:

$$
\varepsilon_{x}=\frac{\sigma_{x}}{E}-\frac{v \sigma_{y}}{E}-\frac{v \sigma_{z}}{E} .
$$

The strains in the $\mathrm{y}$ and $\mathrm{z}$ directions can be obtained in the similar manner:

$$
\begin{aligned}
& \varepsilon_{y}=-v \frac{\sigma_{x}}{E}+\frac{\sigma_{y}}{E}-v \frac{\sigma_{z}}{E} \\
& \varepsilon_{z}=-v \frac{\sigma_{x}}{E}-v \frac{\sigma_{y}}{E}+\frac{\sigma_{z}}{E} .
\end{aligned}
$$

Solving equations (28) and (29) we obtain normal stresses:

$$
\begin{aligned}
& \sigma_{x}=\frac{E}{(1+v)(1-2 v)}\left[\varepsilon_{x}(1-v)+v \varepsilon_{y}+v \varepsilon_{z}\right] \\
& \sigma_{y}=\frac{E}{(1+v)(1-2 v)}\left[v \varepsilon_{x}+(1-v) \varepsilon_{y}+v \varepsilon_{z}\right] . \\
& \sigma_{z}=\frac{E}{(1+v)(1-2 v)}\left[v \varepsilon_{x}+v \varepsilon_{y}+(1-v) \varepsilon_{z}\right]
\end{aligned}
$$

Using $\tau=G \gamma$ we obtain:

$$
\gamma_{x y}=\frac{\tau_{x y}}{G}, \gamma_{y z}=\frac{\tau_{y z}}{G}, \gamma_{z x}=\frac{\tau_{z x}}{G} .
$$


Rearranging equation (31) we have,

$$
\tau_{x y}=G \gamma_{x y}, \tau_{y z}=G \gamma_{y z}, \tau_{z x}=G \gamma_{z x} .
$$

The stresses can be expressed in matrix form

$$
\left\{\begin{array}{l}
\sigma_{x} \\
\sigma_{y} \\
\sigma_{z} \\
\tau_{x y} \\
\tau_{y z} \\
\tau_{z x}
\end{array}\right\}=\frac{E}{(1+v)(1-2 v)}\left[\begin{array}{cccccc}
1-v & v & v & 0 & 0 & 0 \\
v & 1-v & v & 0 & 0 & 0 \\
v & v & 1-v & 0 & 0 & 0 \\
0 & 0 & 0 & \frac{1-2 v}{2} & 0 & 0 \\
0 & 0 & 0 & 0 & \frac{1-2 v}{2} & 0 \\
0 & 0 & 0 & 0 & 0 & \frac{1-2 v}{2}
\end{array}\right],
$$

where,

$$
\begin{gathered}
G=\frac{E}{2(1+v)}, \\
{[D]=\frac{E}{(1+v)(1-2 v)}\left[\begin{array}{cccccc}
1-v & v & v & 0 & 0 & 0 \\
v & 1-v & v & 0 & 0 & 0 \\
v & v & 1-v & 0 & 0 & 0 \\
0 & 0 & 0 & \frac{1-v}{2} & 0 & 0 \\
0 & 0 & 0 & 0 & \frac{1-v}{2} & 0 \\
0 & 0 & 0 & 0 & 0 & \frac{1-v}{2}
\end{array}\right] .}
\end{gathered}
$$

\subsection{Material properties}

In this analysis, material Aluminum, and Steel is used. Here aluminum is used as upper material, steel is used as lower material.

Tab. 1. Properties of the materials used for the analysis

\begin{tabular}{lllll}
\hline Material & $\begin{array}{l}E \\
(\mathrm{Gpa})\end{array}$ & $V$ & $K\left(\mathrm{~W} \cdot \mathrm{m}^{-1} \cdot \mathrm{k}^{-1}\right)$ & $\alpha\left({ }^{\circ} \mathrm{C}\right)$ \\
\hline Aluminium & 72 & 0.33 & 234 & $24 \mathrm{e}-6$ \\
\hline Steel & 210 & 0.3 & 19.5 & $11.6 \mathrm{e}-6$ \\
\hline
\end{tabular}

\subsection{Boundary Conditions}

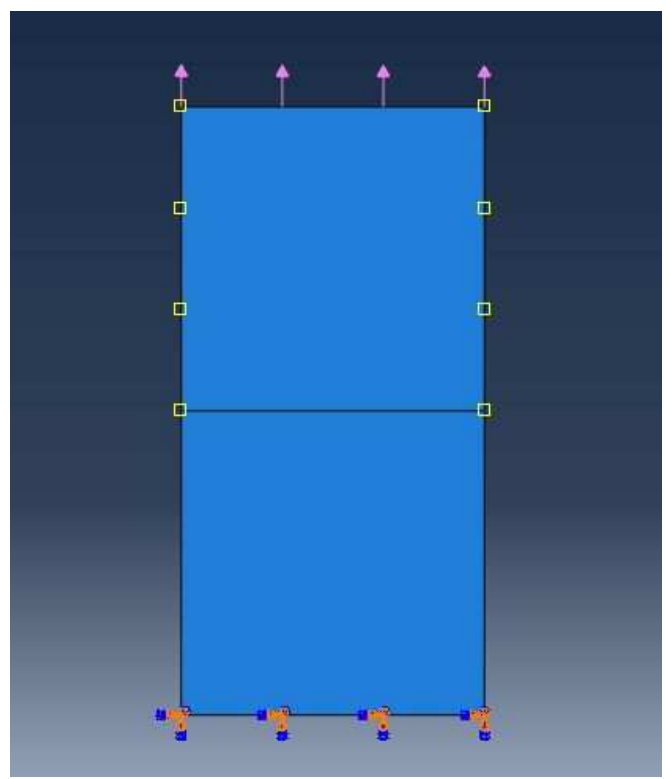

Fig. 3. Model showing boundary condition of the analysis

In this analysis the lower surface is fixed and mechanical load is applied on the upper surface. Left side of the object is at $500^{\circ} \mathrm{C}$ and right side of the object is at $25^{\circ} \mathrm{C}$. After creating the parts and assigning material properties the parts are assembled together. By creating coupled temperature displacment step and applying proper boundary and loading conditions the problem was solved properly.

\section{RESULTS AND DISCUSSIONS}

All simulated results are plotted along the interface to observe stress and displacement characteristics. The graphical illustrations are presented below. 


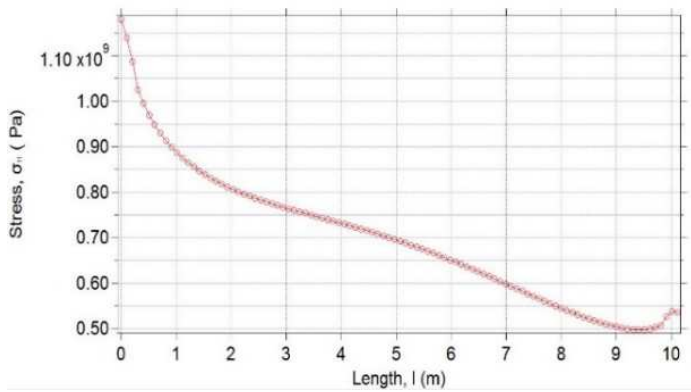

Fig. 4. Variation of $\sigma_{11}$ along the length at the interface of dissimilar material joint

From the Fig. 4 variation of $\sigma_{11}$ along the length at the interface of dissimilar material joint is observed. Since high thermal loadings are applied at the left side of the model high stress developes at the left region and thus satisfying the boundary condition. Very high stress occur at the interface since thermo-mechanical load is applied which may may lead to the delamination initiation in the local area and failure of the joint structures.

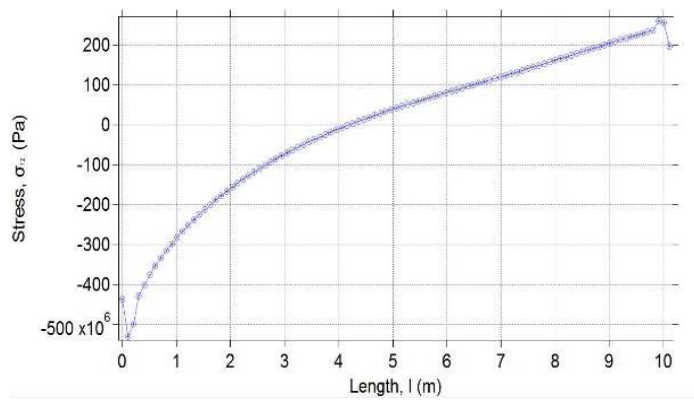

Fig. 5. Variation of $\sigma_{12}$ along the length at the interface of dissimilar material joint

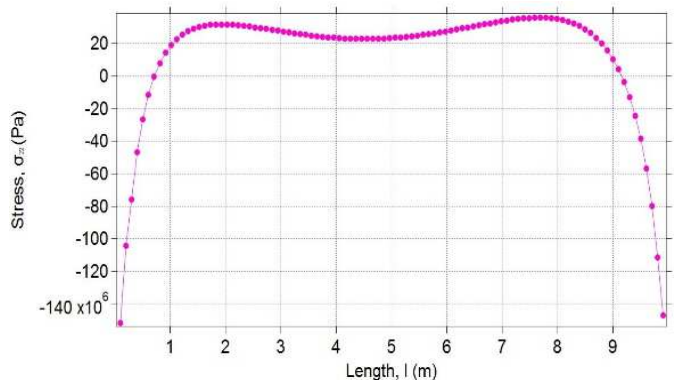

Fig. 6. Variation of $\sigma_{22}$ along the length at the interface of dissimilar material joint
From the Fig. 5, Fig. 6 it is observed that distribution of normal stress $\sigma_{22}$ is symmetric in nature and variation of shear stress $\sigma_{12}$ is anti-symmetric in nature.

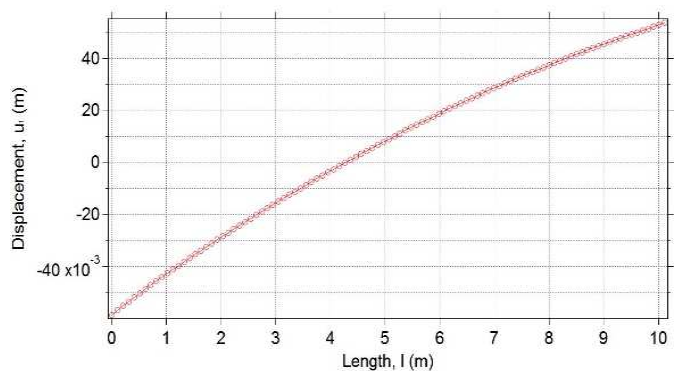

Fig. 7. Variation of $u_{1}$ along the length at the interface of dissimilar material joint

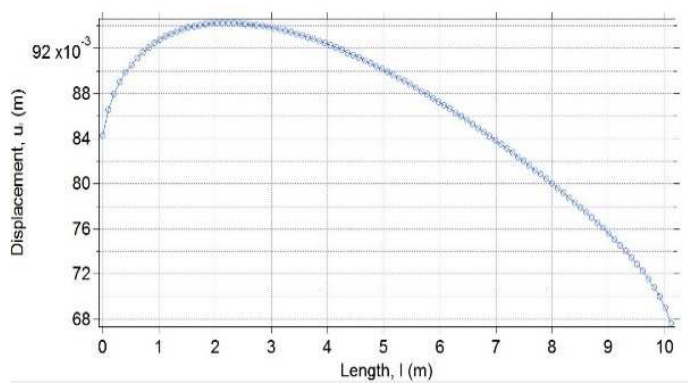

Fig. 8. Variation of $u_{2}$ along the length at the interface of dissimilar material joint

Fig. 7 and Fig. 8. indicates the variation of displacement along the length at the interface of dissimilar material joint. Variation of $\mathbf{u}_{1}$ is quite linear in nature but distribution of $\mathrm{u}_{2}$ varies sharply at the left side region.

From the Fig. 9 it is observed that the common interface of bi-material occurs at $10 \mathrm{~m}$. The illustration indicates the continuity of stress distribution at the interface and thus satisfying the boundary conditions. It also indicates that the joint is reliable and there is no crack at the joint since there is no discontinuity at the interface of dissimilar material joint.

From the Fig. 10 it is observed that the common interface of bi-material occurs at $10 \mathrm{~m}$. The illustration indicates the continuity of displacement distribution at the interface and thus satisfying the boundary conditions. It also indicates that the joint is reliable and there is no crack at the joint since there is no discontinuity at the interface of dissimilar material joint. 

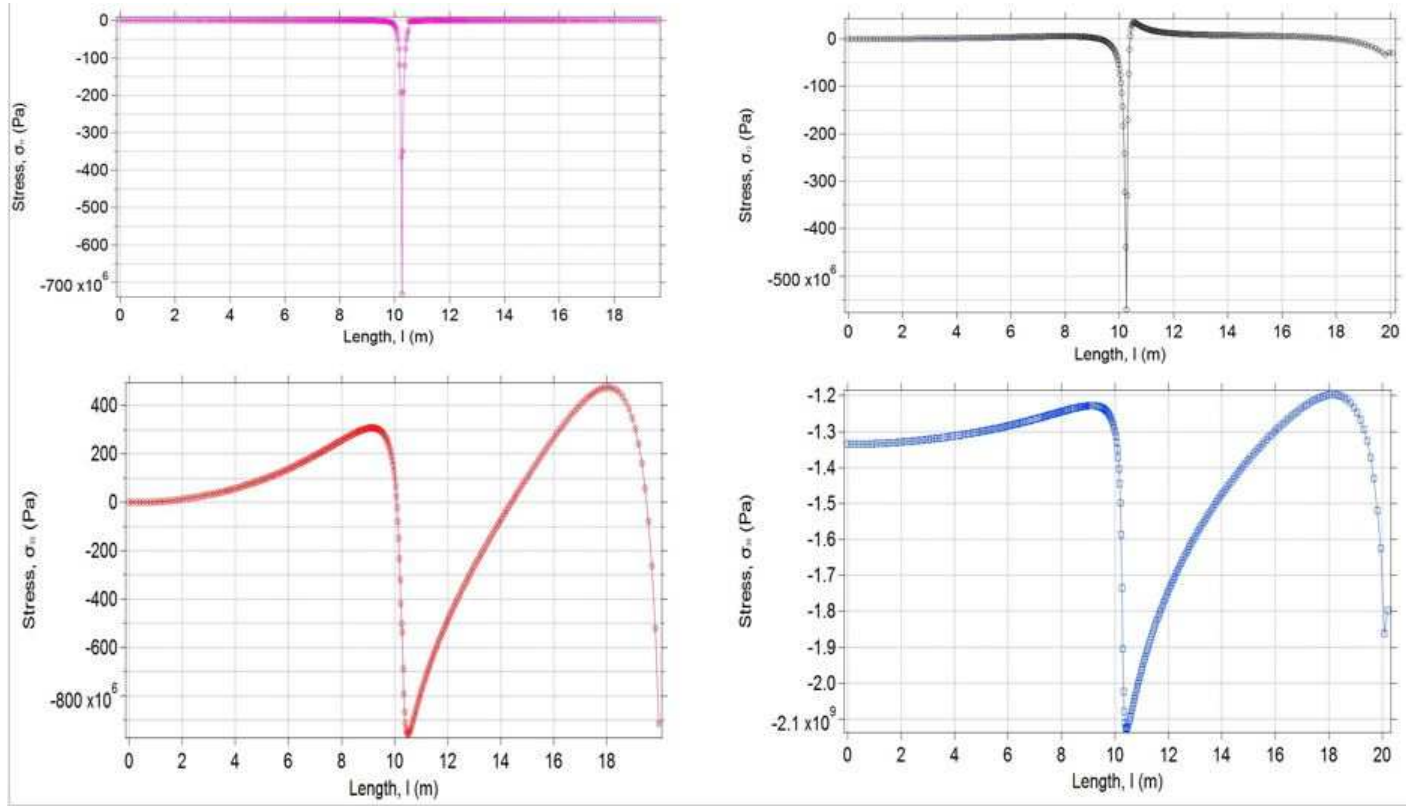

Fig. 9. Variation of stress tensor along the length showing continuity at the interface of dissimilar material joint
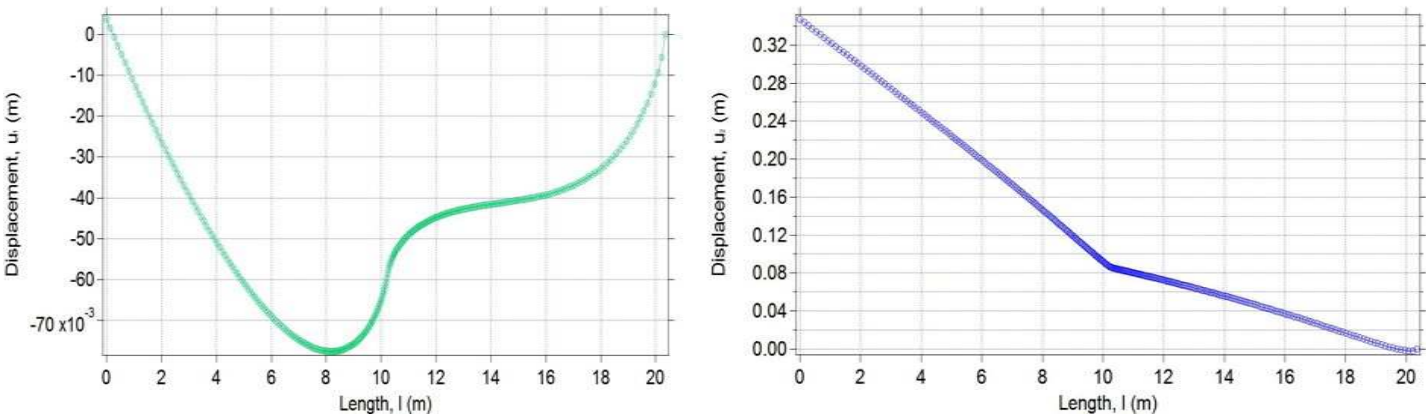

Fig. 10. Variation of displacement tensor along the length showing continuity at the interface of dissimilar material joint

\section{CONCLUSIONS}

The carried out research on the thermo-mechanical stress analysis of dissimilar material using FEM prove that high stress concentration occurs at the interface region than the other regions. Stress continuity is a must for a reliable dissimilar material joint. The normal stress distribution is symmetric in nature but the shear stress distribution is anti-symmetric in nature at the interface of joint. Extra care should be provided at the interface region to prevent failure as high stress concentration occurs at the interface region than the other regions. The thermal loadings have significant effect on the stress distribution at the interface of joint. The combined effects of pressure and temperature must be taken into account when designing dissimilar material joints to ensure reliability of the structure.

\section{Nomenclature}

\section{Symbols}

$\sigma_{\mathrm{xx}}, \sigma_{\mathrm{yy}}, \sigma_{\mathrm{zz}}-$ Normal stress, $\mathrm{Pa}$

$\sigma_{x y}, \sigma_{y z}, \sigma_{z z}-\mathrm{S}$ hear stress, $\mathrm{Pa}$

$\varepsilon_{x}, \varepsilon_{y}, \varepsilon_{z} \quad-\quad$ Normal strain

$\varepsilon_{x y}, \varepsilon_{y z}, \varepsilon_{z x}-$ Shear strain

$E \quad-\quad$ Young modulus, $\mathrm{MPa}$

$G \quad-\quad$ Shear modulus, $\mathrm{MPa}$

$\vartheta \quad-$ Poisson ratio

\section{References}

1. Pahoja H. M (1972) "Stress Analysis of an Adhesive Lap joint Subjected to Tension, Shear Force and Bending Moments", Ph.D. Thesis, University of Illinois, UrbanaChampaign

2. Koguchi H., (1997) "Stress singularity analysis in threedimensional bonded structure", Int. J. Solids Structures, Vol. 34, pp. $461-480$

3. Somadder S., Islam S. (2018) "Stress Analysis of a Cylinder Subjected to Thermo-mechanical Loads by Using FEM",4th Intl. Conf. on Structure, Processing and Properties of Materials, SPPM 2018 
4. Barut A, I. Guven, E. Madenci (2001) "Analysis of singular stress field at joints of multiple dissimilar materials under mechanical and thermal loading", Int. J. Solids Structures, Vol. 38, pp. 9077-9109

5. Koguchi H., Nakajima M., (2010) "Influence of interlayer thickness on the intensity of singular stress field in 3D three-layered joints under an external load", Journal of solid mechanics and materials Engineering, Vol. 4, No 7

6. Koguchi H., Tsukada Y., Kurahashi T. (2013) “Analysis of 3D strain singular field near the corner of SI chip using digital image correlation method", InterPACK2013, Burlingame, CA, USA

7. Luangarpa C., Koguchi H., (2014) "Analysis of a threedimensional dissimilar material joint with one real singularity using a conservative integral", International Journal of Solids and Structures

8. Somadder S., Islam S. (2018) "Stress Analysis of a Thick Walled Orthotropic Bonded Cylinder under Pressure and Temperature by Using FEM" International Journal of Mechatronics and Manufacturing Technology, Vol. 3, Issue 1, pp.1-28

9. Munz D., .Matthais A, Yang Y. Y. (1995) "Thermal stress in ceramic-metal joints with an interlayer", Journal of the American Ceramic Society, Vol. 77, pp. 285-290.

10. Pengfei H., Ishikawa H., Kohno Y. (1995) "Analysis of order of stress singularity at the corner point of a diamond-shape rigid inclusion or hole in an infinite plate bending by conformal mapping", Int. J. Engng. Sci, Vol. 33 , pp. $1535-1546$

\section{Biographical note}

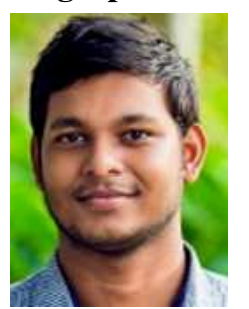

Somnath Somadder received his B. Sc. degree in Mechanical engineering (with honors) from Khulna University of Engineering \& Technology in 2018 (specialization: Computational Solid Mechanics). Currently he is working as a Lecturer in Department of Mechanical Engineering, Khulna University of Engineering \& Technology and doing his M. Sc. degree in same department. This manuscript is made from his research activities as a lecturer during last two years. He has published 2 scientific papers international journals, and conference proceedings .His scientific interest are focused on Analysis of stress singularity fields in 3D transversely isotropic piezoelectric and elastic bonded joints by finite element method. 
